\title{
Beyond the Standard Model searches through $B$ physics at Tevatron
}

\author{
Guennadi Borissov**i \\ Lancaster University, UK \\ E-mail: G.Borissov@lancaster.ac.uk
}

Recent measurements of the Tevatron experiments related to the search for new phenomena beyond the Standard Model are reviewed. The new measurement of $\operatorname{Br}\left(B_{s} \rightarrow \mu^{+} \mu^{-}\right)$reported by the CDF and $\mathrm{D} \varnothing$ collaborations does not show any deviation from the SM expectation. The CDF collaboration presented a new study of the $b \rightarrow s \mu^{+} \mu^{-}$transition which is consistent and competitive with the results from $B$ factories. The CDF collaboration reported the first measurement of the polarization amplitudes in the $B_{s} \rightarrow \phi \phi$ decays. The new measurement of the like-sign dimuon charge asymmetry performed by the $\mathrm{D} \emptyset$ experiment reveals a $3.2 \sigma$ deviation from the SM prediction, while the updated study of the $B_{s} \rightarrow J / \psi \phi$ decay by the CDF and D $\emptyset$ collaboration demonstrates a better agreement with the SM. All experimental results on the $C P$ violation in mixing are currently consistent with each other. Both collaboration have much more statistics to analyze, and all these results can be significantly improved in the future.

35th International Conference of High Energy Physics - ICHEP2010,

July 22-28, 2010

Paris France

\footnotetext{
*Speaker.

$\dagger$ on behalf of CDF and D $\varnothing$ collaborations.
} 
The history of particle physics shows that the first evidence of new phenomena very often comes from the flavour physics. Weak decays of light mesons indicated the existence of the fourth quark; the study of $C P$ violation supported the hypothesis of the third quark family, and the $B^{0}$ meson oscillation lead to the conclusion that the top quark must be heavy. The $B$ hadrons are especially suitable for the searches beyond the Standard Model (BSM). The $b$ quark is expected to have a strong coupling with new particles of many models due to its large mass and is therefore sensitive to the contribution of new physics; $B$ hadrons are abundantly produced at hadron and $e^{+} e^{-}$colliders, and modern experiments have a well developed technique of their selection and study. The $B_{s}$ meson is one of the most promising particle among all $B$ hadrons for the BSM search. The CP violating phenomena related to $B_{s}$ meson are suppressed in the SM, while they can be significantly enhanced in many theoretical models. The large CKM parameters contributing in the processes involving $B_{s}$ meson can only amplify the possible effect.

The Tevatron experiments are very active in the BSM searches through $B$ physics. This paper reviews the new results of $\mathrm{CDF}$ and $\mathrm{D} \varnothing$ collaboration presented at the 35th International Conference of High Energy Physics, such as the search for $B_{s} \rightarrow \mu^{+} \mu^{-}$decay, the study of $b \rightarrow s \mu^{+} \mu^{-}$ transition, the measurement of the like-sign dimuon charge asymmetry, and the study of $B_{s} \rightarrow J / \psi \phi$ and $B_{s} \rightarrow \phi \phi$ decays.

The branching fraction of $B_{s}$ and $B_{d}$ decay to $\mu^{+} \mu^{-}$pair is expected to be very small in the Standard Model [1]:

$$
\begin{aligned}
& \operatorname{Br}\left(B_{d} \rightarrow \mu^{+} \mu^{-}\right)=(1.1 \pm 0.1) \times 10^{-10}, \\
& \operatorname{Br}\left(B_{s} \rightarrow \mu^{+} \mu^{-}\right)=(3.6 \pm 0.3) \times 10^{-9}
\end{aligned}
$$

These values can be significantly modified by the contribution of new physics, and many models predict a much larger branching fraction of $B_{s} \rightarrow \mu^{+} \mu^{-}$decay [2].

The $\mathrm{D} \emptyset$ collaboration presented an update of the $B_{s} \rightarrow \mu^{+} \mu^{-}$search using an integrated luminosity of $6.1 \mathrm{fb}^{-1}$ [3]. This new analysis contains many important improvements comparing to the previous published $\mathrm{D} \emptyset$ results [4]. The muon identification and trigger selection are improved; a Bayesian neural network (BNN) is used instead of the likelihood ratio method, and the upper limit on the branching fraction is calculated in several bins of the BNN output variable $\beta$ and $\mu^{+} \mu^{-}$invariant mass $M\left(\mu^{+} \mu^{-}\right)$. Figure 1 shows the the distribution of $M\left(\mu^{+} \mu^{-}\right)$in the highest sensitivity $\beta$ region (a), and the distribution of $\beta$ in the highest sensitivity $M\left(\mu^{+} \mu^{-}\right)$region (b) for data (dots with uncertainties), expected background distribution (solid line), and the SM signal distribution multiplied by a factor of 100 (dotted-dashed line). The dimuon background contributions from the $B(D) \rightarrow \mu^{+} v X$ decays (dashed line) and the $B \rightarrow \mu^{+} v D X$ decays (dotted line) are also shown. The result of this analysis gives the new upper limit

$$
\operatorname{Br}\left(B_{s} \rightarrow \mu^{+} \mu^{-}\right)<5.1 \times 10^{-8} \text { (observed) },<4.2 \times 10^{-8} \text { (expected), 95\% C.L. }
$$

The CDF collaboration previously also released an update of the $B_{d} \rightarrow \mu^{+} \mu^{-}$and $B_{s} \rightarrow \mu^{+} \mu^{-}$ search using an integrated luminosity of $3.7 \mathrm{fb}^{-1}$ [5]. They obtained

$$
\begin{aligned}
& \operatorname{Br}\left(B_{s} \rightarrow \mu^{+} \mu^{-}\right)<4.3 \times 10^{-8} \text { (observed) },<3.3 \times 10^{-8} \text { (expected), 95\% C.L. } \\
& \operatorname{Br}\left(B_{d} \rightarrow \mu^{+} \mu^{-}\right)<7.6 \times 10^{-9} \text { (observed) },<9.1 \times 10^{-9} \text { (expected), 95\% C.L.. }
\end{aligned}
$$



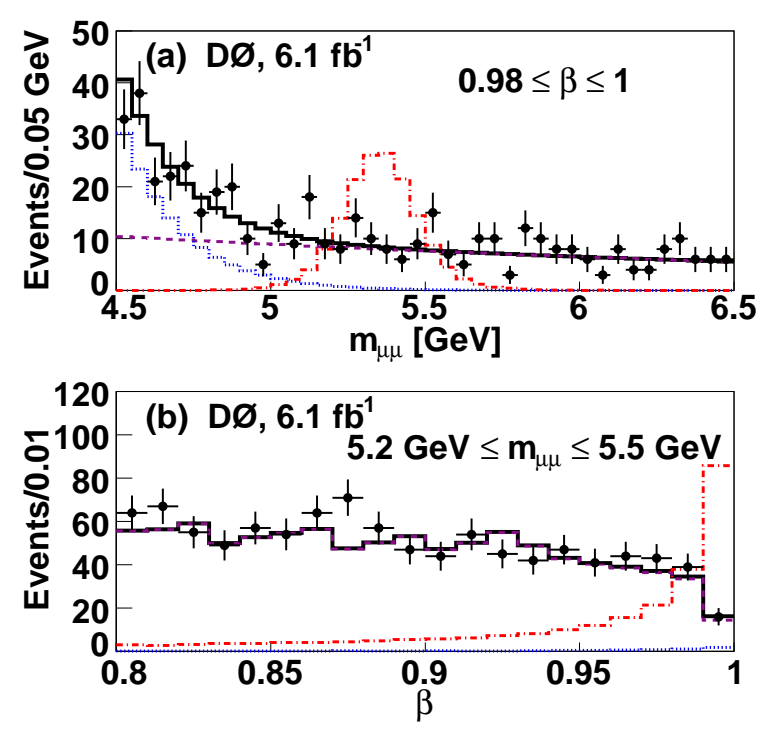

Figure 1: The distribution of $M\left(\mu^{+} \mu^{-}\right)$in the highest sensitivity $\beta$ region (a), and the distribution of $\beta$ in the highest sensitivity $M\left(\mu^{+} \mu^{-}\right)$region (b) for data (dots with uncertainties), expected background distribution (solid line), and the SM signal distribution multiplied by a factor of 100 (dotted-dashed line).

This is the world best upper limit on $\operatorname{Br}\left(B_{s} \rightarrow \mu^{+} \mu^{-}\right)$and $\operatorname{Br}\left(B_{d} \rightarrow \mu^{+} \mu^{-}\right)$.

The upper limit on $\operatorname{Br}\left(B_{s} \rightarrow \mu^{+} \mu^{-}\right)$obtained by the $\mathrm{CDF}$ and $\mathrm{D} \emptyset$ experiments already now constrains the parameters of several new models and will be improved in the future with the increase of statistics. Nevertheless, the Tevatron collaborations will not be able to reach the SM level of $\operatorname{Br}\left(B_{s} \rightarrow \mu^{+} \mu^{-}\right)$and this task will be completed by the LHC experiments.

Another rare decay $b \rightarrow s \mu^{+} \mu^{-}$is also very promising for the BSM searches. This process occurs in the Standard Model only through loop diagrams and is therefore sensitive to the contribution of new physics. This contribution can be detected by comparing the experimental observables like the lepton forward-backward asymmetry or the differential branching fraction as a function of dilepton mass with the SM prediction.

The CDF collaboration presented an update of the analysis of $B^{+} \rightarrow K^{+} \mu^{+} \mu^{-}, B^{0} \rightarrow K^{* 0} \mu^{+} \mu-$ and $B_{s} \rightarrow \phi \mu^{+} \mu^{-}$decays using an integrated luminosity of $4.4 \mathrm{fb}^{-1}$ [6]. The measured branching fractions of these decay modes are found to be

$$
\begin{aligned}
\operatorname{Br}\left(B^{+} \rightarrow K^{+} \mu^{+} \mu^{-}\right) & =0.38 \pm 0.05 \text { (stat) } \pm 0.03 \text { (syst) } \\
\operatorname{Br}\left(B^{0} \rightarrow K^{* 0} \mu^{+} \mu^{-}\right) & =1.06 \pm 0.14 \text { (stat) } \pm 0.09 \text { (syst) } \\
\operatorname{Br}\left(B_{s} \rightarrow \phi \mu^{+} \mu^{-}\right) & =1.44 \pm 0.36 \text { (stat) } \pm 0.46 \text { (syst) }
\end{aligned}
$$

This is the first observation of the $B_{s} \rightarrow \phi \mu^{+} \mu^{-}$decay mode with the statistical significance $6.3 \sigma$. The lepton forward-backward asymmetry $A_{F B}$ in the decay $B^{0} \rightarrow K^{* 0} \mu^{+} \mu^{-}$together with the SM prediction are shown in Fig. 2. The obtained results are consistent and competitive with the other current best results obtained at $B$ factories [7]. At present there is no evidence of discrepancy from the SM prediction [8]. 


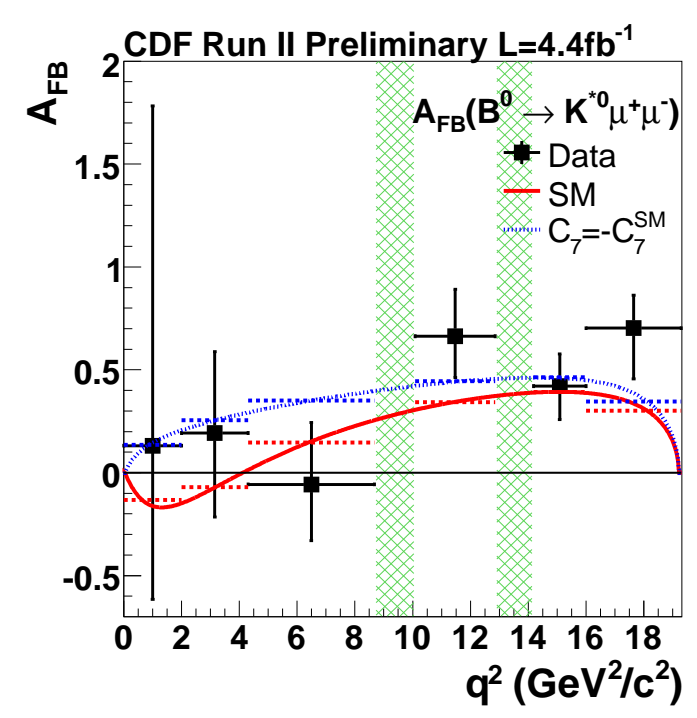

Figure 2: Differential forward-backward asymmetry of $B^{0} \rightarrow K^{* 0} \mu^{+} \mu^{-}$decay as a function of squared dimuon mass. Points are the fit result. The solid curve is the SM expectation [8]. The dotted curve is the $C_{7}=-C_{7}^{\text {eff }}$ expectation, where $C_{7}$ is one of the Wilson coeffcients. The dashed line is the averaged expectation in each squared dimuon mass bin and hatched regions are charmonium veto regions.

One of the promising directions of the search for the new physics is the study of $C P$ violation in mixing of neutral $B$ mesons. The source of this type of $C P$ violation is a phase $\phi_{q}$ of the complex $B_{q}(q=d, s)$ mass matrix. The Standard Model predicts very small values of $\phi_{d}$ and $\phi_{s}$ which are significantly below current experimental sensitivity [9]. The new physics can significantly change them [10]. Therefore, a deviation from zero in these phases would be an unambiguous indication of the BSM contribution. One possibility to constrain the $C P$ violating phase $\phi_{q}$ is to measure the like-sign dimuon charge asymmetry $A_{s l}^{b}$. This quantity is defined as the charge asymmetry in the number of events with two muons of the same charge produced in direct $b \bar{b} \rightarrow \mu \mu X$ decays. In such decays one of muons comes from the decay of oscillating neutral $B$ meson, and the non-zero $C P$ violating phase $\phi_{q}$ results in the non-zero value of $A_{s l}^{b}$. Since both $B_{d}$ and $B_{s}$ mesons are produced in $p \bar{p}$ collisions at the Tevatron, the asymmetry $A_{s l}^{b}$ is sensitive to both the $\phi_{d}$ and $\phi_{s}$ phases.

The D $\varnothing$ collaboration reported a new measurement of $A_{s l}^{b}$ using an integrated luminosity of $6.1 \mathrm{fb}^{-1}$ [11]. In this analysis the events with two muons of the same charge and with one muon are selected, and the inclusive like-sign dimuon and single muon charge asymmetries are measured. In addition to the direct $b \rightarrow \mu X$ decay many other background processes contribute in these samples. Only the direct semileptonic decay $b \rightarrow \mu X$ can produce the $C P$ violating charge asymmetry; the charge asymmetry of all other processes is detector-related. All background contributions in this analysis are determined experimentally with the reduced input from simulation. The sample of events with single muon is used to constrain and control the background. With this approach the systematic uncertainties are considerably reduced.

A unique feature of the $D \emptyset$ experiment is a regular reversal of magnet polarities. Due to this reversal the charge asymmetry due to the muon reconstruction is suppressed and the related 
systematic uncertainty is considerably reduced. The remaining dominant source of background asymmetry comes from the difference in the interaction cross section of positive and negative kaons with the detector material. This asymmetry is measured experimentally.

After subtracting the background asymmetries from the inclusive dimuon charge asymmetry, the resulting like-sign dimuon charge asymmetry is found to be

$$
A_{s l}^{b}=-0.957 \pm 0.251 \text { (stat) } \pm 0.146 \text { (syst) }
$$

. This result differs from the SM prediction by $\sim 3.2 \sigma$. The asymmetry $A_{s l}^{b}$ can be expressed as a linear combination of the semileptonic charge asymmetries $a_{s l}^{q}(q=d, s)$ of $B_{d}$ and $B_{s}$ mesons. Therefore, the obtained result is presented in Fig. 3 as a diagonal band in a two dimensional plane of $a_{s l}^{s}$ versus $a_{s l}^{d}$. This result is in a good agreement with other measurements of the $a_{s l}^{s}$ and $a_{s l}^{d}$ asymmetries $[12,13]$.

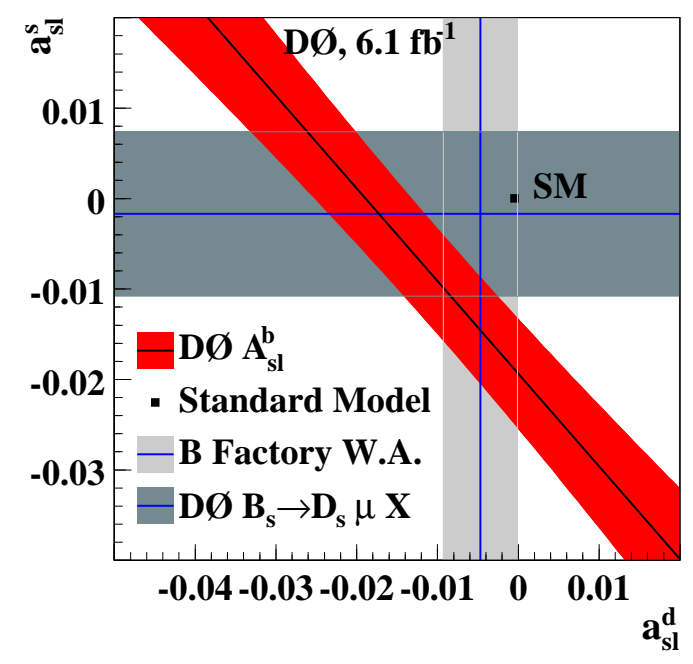

Figure 3: Comparison of $A_{s l}^{b}$ with the standard model prediction for $a_{s l}^{d}$ and and $a_{s l}^{s}$. Also shown are the existing measurements of $a_{s l}^{d}$ [12] and $a_{s l}^{s}$ [13]. The error bands represent the \pm 1 standard deviation uncertainties on each individual measurement.

The decays $B_{s} \rightarrow J / \psi \phi$ offers a complementary possibility to probe the $C P$ violation in mixing of $B_{s}$ meson. The $C P$ violation in this decay is described by the phase $\phi^{J / \psi \phi}$. Within the Standard Model this phase is related with the angle $\beta_{s}$ of the $(b s)$ unitarity triangle and should be very small [9]:

$$
\phi^{J / \psi \phi}=-2 \beta_{s}=2 \arg \left(-\frac{V_{t b} V_{t s}^{*}}{V_{c b} V_{c s}^{*}}\right)=-0.038 \pm 0.02
$$

However, it can be significantly modified by the new physics contribution and its variation is the same as for the phase $\phi_{s}[9]$.

Both the CDF and DØ collaborations presented an update of their $B_{s} \rightarrow J / \psi \phi$ analysis. The CDF collaboration analyzed statistics corresponding to an integrated luminosity of $5.2 \mathrm{fb}^{-1}$ [14]. In total they reconstructed the world largest sample of $B_{s} \rightarrow J / \psi \phi$ decays containing about 6500 
events. The new analysis contains many important improvements, such as the calibration of the same-side flavour tagging using the $B_{s}$ meson oscillation, or addition of $K^{+} K^{-} S$-wave in the fit. Using this sample the CDF collaboration obtained the world most precise measurement of the lifetime and width difference of $B_{s}$ meson:

$$
\begin{aligned}
\tau_{s} & =1.529 \pm 0.025 \text { (stat) } \pm 0.012 \text { (syst) ps } \\
\Delta \Gamma_{s} & =0.075 \pm 0.035 \text { (stat) } \pm 0.01 \text { (syst) } \mathrm{ps}^{-1} .
\end{aligned}
$$

The result of this analysis on the $C P$ violating phase $\beta_{s}=-\phi^{J / \psi \phi} / 2$ is shown in Fig. 4. A good consistency with the SM prediction is observed with the p-value at the Standard Model point is found to be $44 \%$.

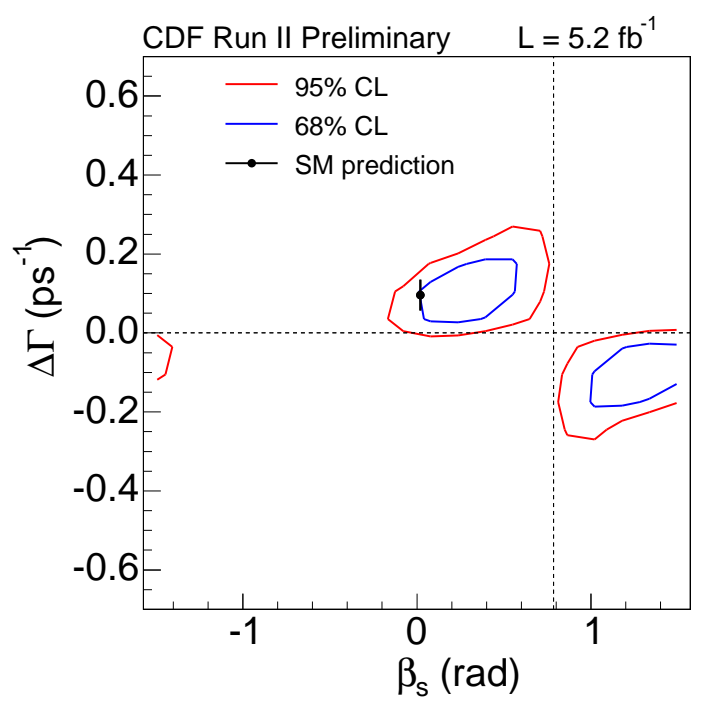

Figure 4: Adjusted two-dimensional profile likelihood of $\beta_{s}$ and $\Delta \Gamma_{s}$. The standard model point is indicated by the black point with error bars.

The $\mathrm{D} \emptyset$ collaboration presented an update of their analysis of $C P$ violation in $B_{s} \rightarrow J / \psi \phi$ decay using an integrated luminosity of $6.1 \mathrm{fb}^{-1}[15]$. They obtained

$$
\begin{aligned}
\tau_{s} & =1.47 \pm 0.04 \text { (stat) } \pm 0.01 \text { (syst) ps } \\
\Delta \Gamma_{s} & =0.15 \pm 0.06 \text { (stat) } \pm 0.01 \text { (syst) } \mathrm{ps}^{-1} .
\end{aligned}
$$

Their result on the $C P$ violating phase $\phi^{J / \psi \phi}$ is shown in Fig. 5. The $68 \%$ C.L. constraint on the phase $\phi_{s}$ derived from the like-sign dimuon charge asymmetry is also shown in Fig. 5. It can be seen that both results are consistent, although the measurement of $\phi_{s}$ phase from $B_{s} \rightarrow J / \psi \phi$ is better consistent with the Standard Model. The CDF and DØ measurements are also consistent with each other.

The decay $B_{s} \rightarrow \phi \phi$ is a complementary channel to study the $C P$ violation involving $B_{s}$ meson. This decay occur through $b \rightarrow s$ penguin diagram, and the comparison of $C P$ violation properties in this decay and in the decay $B_{s} \rightarrow J / \psi \phi$ can be very instructive. The CDF collaboration collected a 


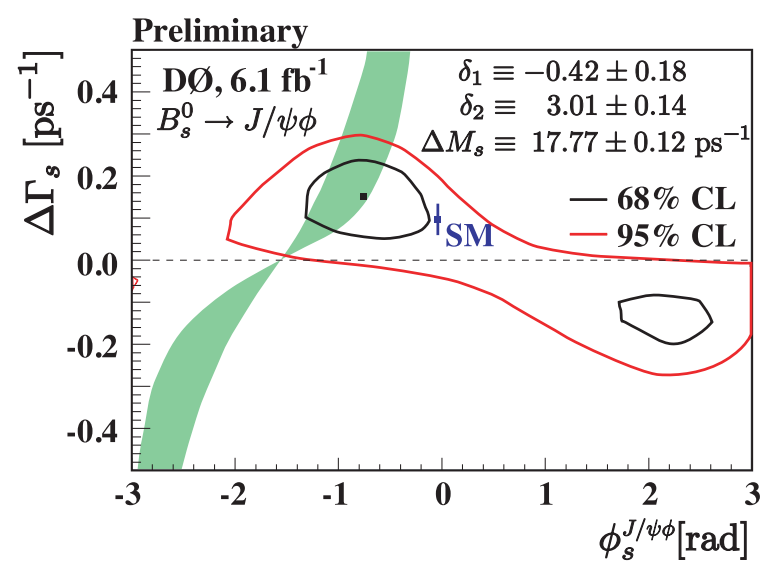

Figure 5: $68 \%$ and $95 \%$ C.L. contours in the plane $\Delta \Gamma_{s}-\phi_{s}$. Also shown is the $68 \%$ contour from the D0 dimuon charge asymmetry analysis [11]. The comparison is made under the assumption of a single source of the CP violation in the $B_{s}-\bar{B}_{s}$ mixing.

large statistics of this decay sufficient to perform the angular analysis. Using the integrated luminosity of $2.9 \mathrm{fb}^{-1}$ they reconstructed about 300 such decays and performed the first measurement of the branching fraction and polarization amplitudes [16]. The measured amplitudes in this decay are found to be

$$
\begin{aligned}
\left|A_{0}\right| & =0.348 \pm 0.041 \text { (stat) } \pm 0.021 \text { (syst) } \\
\left|A_{\|}\right| & =0.287 \pm 0.043 \text { (stat) } \pm 0.011 \text { (syst) } \\
\left|A_{\perp}\right| & =0.365 \pm 0.044 \text { (stat) } \pm 0.027 \text { (syst) }
\end{aligned}
$$

These results shows a large transverse polarization fraction consistent with the theoretical expectations [17].

In conclusion, the Tevatron experiments are very active the searches for new physics beyond the Standard Model. The new results in $B_{s} \rightarrow \mu^{+} \mu^{-}, b \rightarrow s \mu^{+} \mu^{-}$and $B_{s} \rightarrow \phi \phi$ have been presented at the 35th International Conference of High Energy Physics.The new measurement of the like-sign dimuon charge asymmetry performed by the $\mathrm{D} \emptyset$ experiment reveals a $3.2 \sigma$ deviation from the SM prediction, while the updated study of the $B_{s} \rightarrow J / \psi \phi$ decay by the CDF and D collaboration demonstrates a better agreement with the SM. Nevertheless, all experimental results are currently consistent with each other and further study with improved precision is required to reveal a possible contribution of new physics. The uncertainty of all measurements included in this report is statistically dominated, and all results can be improved in the future with the increase of collected statistics.

\section{References}

[1] A. Buras, Prog. Theor. Physics 122, 145 (2009).

[2] S. R. Choudhury and N. Gaur, Phys. Lett. B 451, 86 (1999). J. K. Parry, Nucl. Phys. B 760, 38 (2007). E. Lunghi, W. Porod, and O. Vives, Phys. Rev. D 74, 075003 (2006). D. Guadagnoli, S. Raby and D. 
M. Straub, J. High Energy Phys. 0910, 059 (2009). B. C. Allanach, G. Hiller, D. R. T. Jones and P. Slavich, J. High Energy Phys. 0904, 088 (2009).

[3] V.M. Abazov et al. (D0 Collaboration), Phys. Lett. B 693, 539 (2010).

[4] V.M. Abazov et al. (D0 Collaboration), Phys. Rev. Lett. 94, 071802 (2005).

V.M. Abazov et al. (D0 Collaboration), Phys. Rev. D 76, 092001 (2007).

[5] CDF Collaboration, Public Note 9898.

[6] T. Aaltonen et al. (CDF Collaboration), arXiv:1101.1028 [hep-ex].

[7] B. Aubert et al. (BABAR Collaboration), Phys. Rev. D79, 031102 (2009).

J. T.Wei et al. (BELLE Collaboration), Phys. Rev. Lett. 103, 171801 (2009).

[8] A. Ali, P. Ball, L. T. Handoko, and G. Hiller, Phys. Rev. D61, 074024 (2000).

[9] A. Lenz, U. Nierste, J. High Energy Phys., 0706, 072 (2007).

[10] L. Randall and S. Su, Nucl. Phys. B 540, 37 (1999). J.L. Hewett, arXiv:hep-ph/9803370 (1998). G.W.S. Hou, arXiv:0810.3396 [hep-ph] (2008). A. Soni et al., arXiv:1002.0595 (2010) [hep-ph] and references therein.

[11] V.M. Abazov et al. (D0 Collaboration), Phys. Rev. D 82, 032001 (2010).

[12] E. Barberio et al. (HFAG), arXiv:0808.1297 [hep-ex] (2008).

[13] V.M. Abazov et al. (D0 Collaboration), Phys. Rev. D 82, 012003 (2010).

[14] CDF Collaboration, Public Note 10206.

[15] D0 Collaboration, D0note 6098-CONF.

[16] CDF Collaboration, Public Notes 10064 and 10120.

[17] A. Datta et al. arXiv:0802.0897 [hep-ph] (2008).

M. Beneke, J. Rohrerand and D. Yang, Nucl. Phys. B, 774, 64 (2007). 